\title{
Superfluid-insulator transition of disordered bosons in one dimension
}

\author{
Ehud Altman, ${ }^{1}$ Yariv Kafri, ${ }^{2}$ Anatoli Polkovnikov, ${ }^{3}$ and Gil Refael ${ }^{4}$ \\ ${ }^{1}$ Department of Condensed Matter Physics, The Weizmann Institute of Science, Rehovot 76100, Israel \\ ${ }^{2}$ Department of Physics, Technion, Haifa 32000, Israel \\ ${ }^{3}$ Department of Physics, Boston University, Boston, Massachusetts 02215, USA \\ ${ }^{4}$ Department of Physics, California Institute of Technology, MC 114-36, Pasadena, California 91125, USA
}

(Received 19 November 2009; revised manuscript received 20 April 2010; published 25 May 2010)

\begin{abstract}
We study the superfluid-insulator transition in a one-dimensional system of interacting bosons, modeled as a disordered Josephson array, using a strong-randomness real-space renormalization-group technique. Unlike perturbative methods, this approach does not suffer from run-away flows and allows us to study the complete phase diagram. We show that the superfluid-insulator transition is always Kosterlitz-Thouless like in the way that length and time scales diverge at the critical point. Interestingly however, we find that the transition at strong disorder occurs at a nonuniversal value of the Luttinger parameter, which depends on the disorder strength. This result places the transition in a universality class different from the weak disorder transition first analyzed by Giamarchi and Schulz [Europhys. Lett. 3, 1287 (1987)]. While the details of the disorder potential are unimportant at the critical point, the type of disorder does influence the properties of the insulating phases. We find three classes of insulators which arise for different classes of disorder potential. For disorder only in the charging energies and Josephson coupling constants, at integer filling we find an incompressible but gapless Mott-glass phase. If both integer and half-integer filling factors are allowed then the corresponding phase is a random-singlet insulator, which has a divergent compressibility. Finally in a generic disorder potential the insulator is a Bose glass with a finite compressibility.
\end{abstract}

DOI: $10.1103 /$ PhysRevB.81.174528

PACS number(s): 74.81.-g, 05.30.Jp

\section{INTRODUCTION}

Superfluid-insulator transitions occur in a variety of experimental systems, ranging from low-temperature helium through Josephson arrays to ultracold atomic systems. The simplest paradigm of such a transition is the rather well understood Mott transition of interacting bosons on a perfect lattice commensurate with the boson density. ${ }^{1,2}$ The theoretical picture is far less clear in disordered systems, which occur in a wide variety of experiments: helium in vycor, superconductor-metal, and superconductor-insulator transitions in nanowires and thin films. ${ }^{3-15}$ Recently, disordered systems were also realized using ultracold atoms. ${ }^{16-24}$ Furthermore, this topic was brought back into the limelight with recent experiments in solid helium-4, which show the appearance of a superfluid fraction. ${ }^{25}$ One suggested explanation for this phenomenon is helium turning superfluid in structural defects of the surrounding solid. ${ }^{26}$

Of particular interest is the superfluid-insulator transition in disordered one-dimensional (1D) systems. Even without the disorder the superfluid phase in one dimension is more subtle than in high dimensions. In particular, it does not exhibit true long-range order. Nevertheless the uniform superfluid admits a simple description in terms of a universal harmonic theory or Luttinger liquid. In the opposite limit of a disordered potential but no interactions, particles are always localized. One might naively guess that there is no superfluid phase in the presence of disorder since interaction alone or disorder alone both have a localizing effect on the bosons. This however does not seem to be the case.

The simplest way to see this is to introduce disorder as a perturbation to the interacting superfluid within the Luttinger liquid description. This was done by Giamarchi and Schulz
(GS) in Refs. 27 and 28. The main result of this approach is to describe a phase transition between an essentially uniform superfluid, in which the disorder is irrelevant, into a localized phase. The natural tuning parameter of the transition is the interaction constant and it occurs at a universal value of the Luttinger parameter, independent of the strength of the disorder.

The above approach suffers from two main limitations. First, because it is perturbative in the disorder strength localization is signaled by a runaway renormalization-group (RG) flow. Therefore the approach does not allow for a detailed theory of the insulating phase. Second, the natural regime for the phase transition in this analysis is that of strong interactions and a nearly uniform superfluid, which is not always the case in systems of interest. For example, atom-chip traps, in which ultracold atoms seem to undergo a localization transition, ${ }^{17}$ are in precisely the opposite regime. The bosons are weakly interacting while the potential they feel is highly disordered. ${ }^{29}$ It is not clear whether the analysis of Giamarchi and Schulz provides a valid description of the transition in such a system. Third, within the Giamarchi-Schulz approach it is assumed that disorder always remains narrow and Gaussian and fully characterized by the width. It is unclear that such a distribution is an attractor especially in $1 \mathrm{D}$, where weak links characterizing tails of the distribution can be very important and lead to Griffiths effects.

Different approaches have been used to specifically describe the insulating phases of bosons and suggested several possibilities depending on the nature of the system. In the most generic disordered potential, Ref. 30 argued for the formation of a Bose-glass phase characterized by a finite compressibility and diverging local superfluid susceptibility. In the presence of a commensurate lattice Refs. 31 and 32 
predicted the existence of a Mott-glass phase, an incompressible yet gapless insulator.

In recent work we introduced a unified approach to treat both the phase transition at strong disorder as well as the properties of the insulating phases. ${ }^{33,34}$ For this purpose we employed a real-space renormalization-group (RSRG) technique. ${ }^{35-38}$ We found that the superfluid-insulator transition at strong disorder is insensitive to the type of disorder introduced into the system. It is always Kosterlitz-Thouless like in the following sense: characteristic time scales and length scales both diverge at the transition as $\exp \left(1 / \sqrt{\alpha-\alpha_{c}}\right)$, where $\alpha$ is the tuning parameter. The nature of the disordered superfluid phase is also universal. It is described by an effective harmonic chain with random Josephson couplings drawn from universal distributions generated as fixed points of the RSRG flow. These distributions were recently used to compute the localization behavior of density waves. ${ }^{39}$

The symmetry properties of the disorder, while not important in the superfluid phase or the transition, are crucial for determining the nature of the insulating phases. Using the RSRG approach we confirmed the formation of a Bose-glass phase for generic disorder and a Mott glass for a commensurate lattice with off-diagonal disorder. The latter phase was also seen in recent numerical simulations ${ }^{40,41}$ (numerical confirmations of the existence of the Bose-glass date further back, e.g., Refs. 42 and 43). In addition we found a glassy phase, which we termed a random-singlet glass, in a system with particle-hole symmetry. This phase is characterized by a divergence of both compressibility and superfluid susceptibility. Nevertheless it is still insulating, with conductance dropping as $\exp (-\sqrt{L})$ with length. This phase is analogous to the random-singlet phase found in the spin- $\frac{1}{2} \mathrm{X}-\mathrm{Y}$ chain. ${ }^{36}$

The purpose of the present paper is twofold. First, we provide the detailed analysis of chains with generic diagonal disorder, leading to the results of Ref. 34. Second, we extend the analysis and compute the value of the Luttinger parameter at the phase transition within the RSRG method. We find that, at strong disorder, the transition occurs at a nonuniversal value of the Luttinger parameter that depends of the strength of disorder. This is contrary to the perturbative analysis of Refs. 27 and 28.

The structure of the paper is as follows. In Sec. II we define the model we study and discuss its relevance to actual physical systems. We give a detailed derivation of the RSRG flow equations for the special case of particle-hole symmetric disorder in Sec. III and for generic disorder in Sec. IV. We give a detailed account of the numerical as well as the approximate analytical solutions of the flow equations. Then in Sec. V we solve for the value of the Luttinger parameter at the transition. Finally we conclude with a summary of the results and their validity and a discussion of their possible experimental implications.

\section{MODEL}

Our starting point for the theoretical analysis is the quantum rotor Hamiltonian,

$$
\mathcal{H}=\sum_{j} \frac{1}{2} U_{j}\left(\hat{n}_{j}-\bar{n}_{j}\right)^{2}-\sum_{j} J_{j} \cos \left(\varphi_{j+1}-\varphi_{j}\right) .
$$

This model describes an effective Josephson-junction array with random Josephson coupling $J_{i}$ and charging energies $U_{i}$.
In addition there is a random offset charge $\bar{n}_{i}$ to each grain, which is tantamount to a random gate voltage. Although the model can be visualized as a Josephson-junction array, it actually provides an effective description valid for a wide variety of systems that undergo a superfluid to insulator transition. In bosonic systems, in particular, such transitions are usually driven by quantum phase fluctuations. Hamiltonian (1) should then be thought of as a low-energy effective theory one obtains after integrating out the gapped amplitude fluctuations. The remaining degrees of freedom relevant to the transition are the quantum rotors.

One concrete example of how such a model is naturally generated at low energies is provided by a system of ultracold atoms in an atom-chip trap. In this system the disordered potential is induced by corrugation in the wire that generates the trapping magnetic field. ${ }^{29}$ With increasing corrugation, the atoms concentrate in small puddles at minima of the potential. Neighboring puddles are connected with each other by a random Josephson coupling which depends on the potential barrier between them. The result is exactly the random Josephson array defined in Eq. (1).

Another possible physical realization of model (1) is a disordered superconducting nanowire. Here the issue is more subtle because there may be gapless fermionic degrees of freedom that generate dissipation. Indeed Refs. 44 and 45 applied the RSRG to such wires starting from a Hertz-Millis ${ }^{46,47}$ dissipative action, with a dissipation term $|\omega| \psi^{*} \psi$. An alternative approach is to use phase only models, which describe resistively shunted Josephson-junction arrays. ${ }^{48}$ This naturally leads to a dissipation term of the form $q^{2}|\omega| \phi^{*} \phi$, which does not affect global superconductorinsulator transitions. Such models combined with strong disorder may also be described by the present analysis in parts of their phase diagram. Note that we do not discuss here the case of low filling weakly interacting bosons, as in, e.g., Refs. 49 and 50.

\section{PARTICLE-HOLE SYMMETRIC CHEMICAL POTENTIAL DISORDER}

Of all the random coupling constants in model (1), the random offset charge (or local chemical potential) seems to be the hardest to incorporate in an RG treatment. Since the offsets simply add up to give the offset of a block of sites, it seems clear that this disorder will just grow as the square root of the scale of the real-space RG making it hard to track. However this difficulty turns out to be largely superficial and can be easily overcome in the analysis. To make the discussion more transparent we start from the case where only integer and half-integer offset charges, $\bar{n}_{i}$, are allowed on each site. This condition maintains particle-hole symmetry and therefore still does not correspond to the generic case. Nevertheless, this restriction allows a relatively simple RG analysis which affords important analytic and numerical insights into the possible phases and the phase transitions. In the next section we generalize our treatment to the case of generic disorder.

We note that despite the restrictive condition, allowing only $\bar{n}_{j}=0,1 / 2$, this type of disorder may actually be a rea- 
sonable approximation for chains of superconducting grains with pairing gap much larger than the charging energy. Under these conditions we can assume that the electrons on the grains are always paired and we can take $e^{*}=2 e$ as the unit of the bosonic charges. On the other hand the positive background charge is a random number that could be even or odd in units of $e$ and consequently either integer or half integer in units of the boson charge $e^{*}$. Allowing for charged impurities on the substrate or unscreened coulomb interactions between different grains would of course lead violation of the restrictions on the offset charges.

\section{A. Particle-hole symmetric quantum rotor model}

The essence of the renormalization-group transformations either in real or momentum space is the gradual coarse graining of the system. In this section we extend the decimation scheme of Ref. 33 to the Hamiltonian in Eq. (1) for the case that $\bar{n}_{j}$ can take the values of 0 and $1 / 2$ randomly. The last condition ensures the particle-hole symmetry in the problem: the Hamiltonian does not change under the transformation $n \rightarrow 1-n$. These two values of $\bar{n}_{j}$ represent the two possible extremes which drive the physics of the Bose glass. ${ }^{30}$ Sites with $\bar{n}_{j}=0$ have a well-defined Coulomb blockade with charging energy $U_{j}$. Sites with $\bar{n}_{j}=1 / 2$, on the other hand, have no Coulomb blockade. With no further interactions, these sites yield both infinite compressibility and infinite superfluid susceptibility due to the number fluctuations costing no energy. Hamiltonian (1) is characterized by the distribution of hoppings $J_{j}$ and charging energies $U_{j}$, and of the proportion of sites with $\bar{n}=1 / 2$. We will refer to the latter sites as half-integer sites or "half sites."

\section{B. Extended real-space renormalization group}

Let us now construct the extended decimation scheme for model (1). Following Refs. 33 and 35-37 we construct an RG scheme that eliminates iteratively large energy scales from the Hamiltonian. Two sites connected by the strongest bond will be converted to a phase-coherent cluster. Similarly, in sites with strong charging energy $U$ we eliminate all the excited states. However, the result of this elimination will be different for integer and half-integer sites. Let us now discuss these steps in detail.

We denote the largest energy scale in Hamiltonian (1) $\Omega$ $=\max \left\{J_{i}, U_{i}\right\}$. In each step in the RG we eliminate the strongest coupling from the Hamiltonian and hence successively reduce $\Omega$. If the strongest coupling is the charging energy of site $i, U_{i}$, we eliminate all the excited states of this site. For integer sites with $\bar{n}_{i}=0$, we minimize the charging energy by setting $n_{i}=0$ and include the coupling of this site to the rest of the chain perturbatively. As in Ref. 33, the second-order perturbation theory leads to a coupling between the new nearest neighbors $i-1$ and $i+1$,

$$
\widetilde{J}_{i-1, i+1}=J_{i-1} J_{i} / \Omega \text {. }
$$

On the other hand, if $\bar{n}_{i}=1 / 2$ we reduce site's $i$ Hilbert space to the states $n_{i}=0,1$. The hoppings connecting site $i$ to its neighbors are still active and to the first approximation are not affected by the elimination of the high-energy states. The decimation step for $\bar{n}_{i}=1 / 2$ produces a new kind of site, a doublet site, only capable of having $n_{i}=0$ or 1 . Let us denote the fraction of doublet sites as $s$, the fraction of integer sites as $q$, and the fraction of half sites as $p$. Note that these fractions add up to unity $p+q+s=1$.

When the strongest coupling in the chain is the bond $J_{i}$, unless both sites $i$ and $i+1$ are already-decimated doublet sites, a phase-coherent cluster forms. Since charging energy is the inverse of capacitance, the effective $U_{i, i+1}$ of the new cluster will be

$$
\frac{1}{\tilde{U}_{i}}=\frac{1}{U_{i}}+\frac{1}{U_{i+1}} .
$$

For a doublet site, $U_{i}$ is set to $\infty$. It is easy to see that the filling factor $\bar{n}$ is an additive quantity,

$$
\widetilde{\bar{n}}_{i, i+1}=\left(\bar{n}_{i}+\bar{n}_{i+1}\right) \quad \bmod 1 .
$$

Therefore two half sites or two integer sites form an integer cluster. An integer site and a half site form a half cluster. Similarly, a doublet site and an integer site form a half cluster, and a doublet and half site form an integer cluster.

It is important to note here that the above decimation step does not assume long-range order; it states that phase fluctuations within the newly formed cluster are harmonic and therefore the cluster cannot be broken due to phase slips. These harmonic fluctuations are crucial for the understanding of the properties of the superfluid phase, as explained in Sec. V. Nevertheless these phase fluctuations can be neglected for the purpose of the RG flow and they do not change the critical properties of the model. ${ }^{33}$

A qualitatively new decimation step, which goes beyond Ref. 33 occurs when the strong bond $J_{i}$ connects two doublet sites. In this case the two sites form a unique nondegenerate ground state,

$$
\left|\psi_{i, i+1}\right\rangle=\frac{\left|n_{i}=0, n_{i+1}=1\right\rangle+\left|n_{i}=1, n_{i+1}=0\right\rangle}{\sqrt{2}},
$$

which has energy $-J_{i} / 2$. The second-order perturbation theory leads to an effective hopping between sites $i-1$ and $i+2$,

$$
\widetilde{J}_{i-1, i+1}=J_{i-1} J_{i+1} / J_{i}=J_{i-1} J_{i+1} / \Omega .
$$

Since each doublet site can be thought of as a spin-1/2 degree of freedom, the elimination of $J_{i}$ consists of the formation of a singlet. Hence we recover the Ma-Dasgupta RG transformation. ${ }^{35,36}$ Note that formally Eqs. (2) and (6) are identical.

\section{Flow equations}

Next, we describe the flow equations implied by the above decimation steps. As in Refs. 36 and 37, we parametrize the cutoff energy scale with the variable $\Gamma$ $\equiv \log \left(\Omega_{0} / \Omega\right)$, where $\Omega_{0}$ is the initial cutoff. Also, we define the dimensionless couplings $\zeta_{i}=\Omega / U_{i}-1$ which are characterized by probability distributions $f_{q}(\zeta, \Gamma)$ for integer sites 
and $f_{p}(\zeta, \Gamma)$ for half sites. In principle these distributions can be different, but one can show that their difference is irrelevant in the RG sense, and therefore does not affect any of our conclusions. For simplicity, we assume from the beginning that $f_{q}=f_{p} \equiv f$. We also define $\beta_{i}=\log \left(\Omega / J_{i}\right)$ as the logarithmic bond variable, with distribution $g(\beta, \Gamma)$. Note that by construction $\beta_{i}$ and $\zeta_{i}$ have nonzero probability distribution in the interval $[0, \infty)$.

Renormalization-group steps gradually decrease the number of remaining sites in the chain $[N(\Gamma)]$. Thus decimation of the integer site with large charging gap $U$ reduces $N$ by one while a similar decimation of a half-integer site simply converts it to the doublet site. Also, decimation of a strong link and joining two sites into a cluster reduces the number of active sites by one unless the link connects two doublets. In the latter case the number of remaining sites is reduced by two. Thus the flow of $N$ is given by

$$
d N(\Gamma)=-\left[g_{0}(\Gamma)\left(1+s^{2}\right)+q f_{0}(\Gamma)\right] N(\Gamma) d \Gamma,
$$

where $f_{0}(\Gamma) \equiv f(0, \Gamma)$ and $g_{0}(\Gamma) \equiv g(0, \Gamma)$. From Eq. (7) and the above RG conditions, we obtain the flows of the fractions $p, q$, and $s$,

$$
\begin{gathered}
\frac{d s}{d \Gamma}=-g_{0} s\left(1-s^{2}\right)+f_{0}(p+q s), \\
\frac{d p}{d \Gamma}=-g_{0}\left[p\left(1-s^{2}\right)-2 q(1-q)\right]-f_{0}(p-p q), \\
\frac{d q}{d \Gamma}=-g_{0}\left[s^{2}-1+3 q-2 q^{2}-q s^{2}\right]-f_{0}\left(q-q^{2}\right) .
\end{gathered}
$$

It is easy to check that $d s / d \Gamma+d p / d \Gamma+d q / d \Gamma=0$, provided that $p+q+s=1$.

The RG conditions also lead to master equations for the distributions,

$$
\begin{aligned}
\frac{\partial f(\zeta)}{\partial \Gamma}= & (1+\zeta) \frac{\partial f(\zeta)}{\partial \zeta}+(1-s) g_{0} \iint d \zeta_{1} d \zeta_{2} f\left(\zeta_{1}\right) f\left(\zeta_{2}\right) \\
& \times \delta\left(\zeta_{1}+\zeta_{2}+1-\zeta\right)-f(\zeta) g_{0}(1-s)+f(\zeta)\left(f_{0}+1\right), \\
\frac{\partial g(\beta)}{\partial \Gamma}= & \frac{\partial g(\beta)}{\partial \beta}+\left(s^{2} g_{0}+q f_{0}\right) \iint d \beta_{1} d \beta_{2} g\left(\beta_{1}\right) g\left(\beta_{2}\right) \\
& \times \delta\left(\beta_{1}+\beta_{2}-\beta\right)+g(\beta) g_{0}\left(1-s^{2}\right)-q g(\beta) f_{0} .
\end{aligned}
$$

Even though these equations look quite complicated, the meaning of the each term is straightforward. For example, the second term in the first of these equations corresponds to renormalization of the capacitance of the cluster following the decimation of the link. The multiplier $1-s$ reflects the fact that the renormalization takes place only if the link does not connect two singlets.

Equations (8) and (9) can be significantly simplified noting that $p=q=(1-s) / 2$ is their solution for arbitrary functions $f_{0}(\Gamma)$ and $g_{0}(\Gamma)$. It is easy to check that $p=q$ is in fact an attractive solution. Indeed Eq. (8) gives

$$
\frac{1}{q-p} \frac{d(q-p)}{d \Gamma}=-f_{0}(1-q)-g_{0}[2(1-q)+s(1-s)]
$$

Unless $q=1$, the right-hand side of Eq. (10) is always negative, which means that the line $p=q$ is an attractor. Physically one can understand this result as follows: the integer versus half-integer filling of a cluster is determined by the parity of the total number of half-integer decimated sites. As clusters grow in size under RG due to coarse graining, the number of such sites becomes large, and thus even and odd parities occur with the same probability. The case of commensurate disorder, $q=1$, which was analyzed in Ref. 33 is an exception since it corresponds to the strictly zero fraction of half sites where the clusters always remain even.

The other important observation is that in the weakly interacting regime $f_{0} \ll 1$ one can use a simple exponential ansatz to solve Eq. (9),

$$
f(\zeta)=p f_{0} e^{-f_{0} \zeta} \quad g(\beta)=g_{0} e^{-g_{0} \beta} .
$$

As we will see below (see also Ref. 33), the universal properties of the superfluid-insulator transition are determined by the noninteracting fixed point with vanishing $f_{0}$, where the ansatz is well justified. According to our numerical simulations, these exponential scaling functions are attractors of the flow equations and they describe very well the distribution of $\zeta$ and $\beta$ even when $f_{0}$ is not very small (see discussion below). Substituting the ansatz [Eq. (11)] and $p=q=(1-s) / 2$ into Eqs. (8) and (9) we find

$$
\begin{aligned}
& \frac{d f_{0}}{d \Gamma}=f_{0}\left[1-g_{0}(1-s)\left(1+f_{0}\right)\right], \\
& \frac{d g_{0}}{d \Gamma}=-\frac{g_{0}}{2}\left[(1-s) f_{0}+2 s^{2} g_{0}\right], \\
& \frac{d s}{d \Gamma}=\frac{f_{0}}{2}\left(1-s^{2}\right)-g_{0} s(1-s) .
\end{aligned}
$$

This system has two fixed points for $s: s=0$ and $s=1$. The first fixed point $s=0, p=q=1 / 2$ as we will see below describes the superfluid phase while the second one $s=1, p=q=0$ corresponds to the random-singlet glass insulator.

\section{Superfluid (SF) fixed point}

Let us first address the superfluid fixed point $-s=0$ (no doublet sites). Note that from the last equation of the system [Eq. (12)], this fixed point is stable only when $f_{0}$ is small. Since $f_{0}$ flows either to zero or to infinity, the fixed point $s$ $=0$ is stable when $f_{0} \rightarrow 0$. Then the linearized flow equations to first order in $s$ and $f_{0}$ reduce to

$$
\frac{d f_{0}}{d \Gamma}=f_{0}\left(1-g_{0}\right)
$$




$$
\frac{d g_{0}}{d \Gamma}=-\frac{1}{2} f_{0} .
$$

Remarkably, apart from a factor of $1 / 2$ in the second equation, these are the same flow equations as obtained near the SF fixed point of the random Bose-Hubbard model with no half sites (i.e., $q=1$ ), as in Eq. (6) of Ref. 33. The extra factor of $1 / 2$ can be absorbed in a redefinition of $f_{0}$. This factor appears because half of the sites in the lattice have halfinteger filling and hence their interactions are ineffective in suppressing the superfluidity and thus do not renormalize $g_{0}$ which is related to SF stiffness (see Sec. V).

Equations (13) and (14) are easily solved to give

$$
f_{0}(\Gamma)=A+\left[1-g_{0}(\Gamma)\right]^{2},
$$

where $A$ is a tuning parameter that controls the flow. When $A<0$, the flows terminate on the noninteracting fixed line $f_{0}=0, g_{0}>1$. The point $A=0$ lies on the critical manifold, which terminates in a $K-T$ fixed point (note that if we use $v=\sqrt{f_{0}}$ we obtain the standard Kosterlitz-Thouless flow equations) at $g_{0}=1, f_{0}=0$.

At the critical manifold we can solve exatly for the flow of $g_{0}$ and $f_{0}$. Using the parametrization $g_{0}=1+\delta g . f_{0}$ and $\delta g$ are determined by the differential equations,

$$
\begin{gathered}
\frac{\partial \delta g}{\partial \Gamma}=-\frac{1}{2} f_{0}, \\
\frac{\partial f_{0}}{\partial \Gamma}=-f_{0} \delta g .
\end{gathered}
$$

By dividing the two equations we have

$$
d(\delta g)^{2}=d f_{0}
$$

and

$$
\frac{\partial \delta g}{\partial \Gamma}=-\frac{1}{2} \delta g^{2}
$$

This gives

$$
\delta g=\frac{2}{\Gamma}+\frac{2}{\Gamma^{2}} \quad f_{0}=\frac{4}{\Gamma^{2}} .
$$

Note that the solution of the flow equations in the case of only integer sites $(q=1)$ yields the same results, only with $f_{0} \rightarrow 2 / \Gamma^{2}$.

When $A>0$, the parameters $f_{0}$ and $g_{0}$ flow past the fixed point, where $f_{0}$ begins to increase. The increase in $f_{0}$ entails a flow away from the $s=0$ fixed point toward the $s=1$ fixed point. In Fig. 1 we show the examples of flows of $s$ near the critical point both in the superfluid and in the insulating regimes.

We now want to stress a very important point. It appears that in the superfluid regime the system flows to the classical fixed line, where there is no charging term. However, as we hinted above (see Sec. III B), this statement should be understood with special care. Each time the RG scheme merges two sites into a single superfluid cluster it neglects the harmonic Josephson plasmon between the two sites, although its

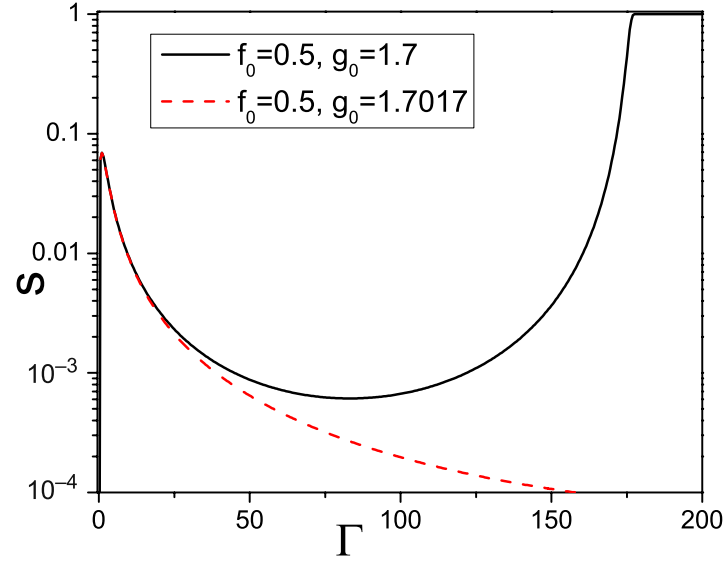

FIG. 1. (Color online) RG flow of the fraction of the singlet sites $s$ according to Eq. (12) near the critical point. The solid line corresponds to the insulating regime while the dashed line does to the superfluid phase. In both cases we assumed there are no singlet sites at the onset, i.e., $s(\Gamma=0)=0$.

energy is well below the RG cutoff at this step. These internal excitations do not influence the progression of the $R G$ flow. However, they become the elementary phonon excitations of the single superfluid cluster that evolves to be the RG fixed point. Therefore the fact that the charging term becomes irrelevant simply means that one should keep only these harmonic phonons. In other words, one can ignore vortices or phase slips, which destroy the superfluid phase if they proliferate. Indeed there is a direct connection between onsite interactions and phase slips. As we show next, at strong disorder, such sites are responsible for renormalization of the superfluid stiffness $\rho_{s}$ playing the role of phase slips. The fact that $f_{0}$ flows to zero implies that such events renormalizing $\rho_{s}$ become unimportant and one can use a noninteracting quadratic description. This issue will be discussed thoroughly in Sec. V. Technically the fact that interactions are irrelevant in our description comes from the fact that we are working in the grand-canonical ensemble. While in the insulating regime there is no difference between excitation energies in canonical and grand-canonical ensembles and in the superfluid regime there is a significant difference. Thus in canonical ensemble the lowest energy excitation corresponds to a phase twist or phonon while in the grand-canonical ensemble the lowest energy corresponds to the addition of an extra particle, which costs much less energy than the phase twist. So the fact that in our scheme the interactions are irrelevant in the SF phase should be understood only in this grand-canonical sense.

\section{E. Insulating fixed point-Random-singlet glass}

The $s=1$ fixed point corresponds to the insulating phase. Indeed one can check that in this limit $g_{0}(\Gamma) \rightarrow 0$ and $f_{0}(\Gamma)$ $\rightarrow \infty$. However, this insulator is not the Mott glass that describes the case of integer only sites considered in Ref. 33. At $s=1$, all the sites remaining in the system are doubly degenerate. These sites can be thought of as spin-1/2 degrees of freedom, with $|\uparrow\rangle=|\bar{n}+1 / 2\rangle$ and $|\downarrow\rangle=|\bar{n}-1 / 2\rangle$. Without 
hopping, the ground state obviously has a huge degeneracy. However, the residual hopping lifts this degeneracy. In the spin language, the hopping terms correspond to usual $x y$ spin-spin interaction,

$$
J_{i} \cos \left(\phi_{i+1}-\phi_{i}\right) \rightarrow J_{i}\left(\sigma_{i}^{x} \sigma_{i+1}^{x}+\sigma_{i}^{y} \sigma_{i+1}^{y}\right) .
$$

Thus we arrive at a spin- $1 / 2$ system with random $x y$ couplings. The ground state of this system is known to be the random-singlet phase. ${ }^{36}$ A strong bond between sites $i$ and $i+1$ delocalizes a boson between two sites and creates a cluster that has a charge gap $J_{i}:\left|\psi_{i, i+1}\right\rangle=\left|1_{i}\right\rangle\left|0_{i+1}\right\rangle+\left|0_{i}\right\rangle\left|1_{i+1}\right\rangle$. Quantum fluctuations produce an effective coupling between sites $i-1$ and $i+2$ as in Eq. (2). The typical length over which the singlets form, $\ell$, scales as $\Gamma^{2}$. Alternatively, one can say that the gaps of each singlet cluster is $\Delta=\Omega_{0} \exp (-\sqrt{\ell})$.

The flow equations can be linearized near $s=1$ and we obtain

$$
\begin{gathered}
\frac{d f_{0}}{d \Gamma}=f_{0}, \\
\frac{d g_{0}}{d \Gamma}=-g_{0}^{2}, \\
\frac{d s}{d \Gamma}=(1-s) f_{0} .
\end{gathered}
$$

The first equation implies that $f_{0}$ diverges as $f_{0} \sim e^{\Gamma}$. This scaling implies that interaction in the remaining nonsinglet sites is narrowly distributed near the maximum energy scale $\Omega$. Following from that scaling of $f_{0}, s$ converges to 1 extremely fast: $s=1-\delta \exp \left(-e^{\Gamma}\right)$. And finally $g_{0}$, which corresponds to the average of $\ln \left(J_{j}\right)$, follows the random-singlet scaling and flows slowly to zero as $g_{0}=\frac{1}{\Gamma}$.

The random-singlet glass is an insulator with the superfluid stiffness of a chain of length $L$ scaling as the spin susceptibility of a random Heisenberg model, as analyzed in Ref. 51,

$$
\rho_{s} \sim e^{-C \sqrt{L}}
$$

with $C$ being a nonuniversal constant (note that for the random Heisenberg chain the susceptibility is the same in all directions). This behavior of $\rho_{s}$ immediately follows from Eqs. (7) and (21), see also Ref. 36, and Ref. 52 where this quantity was studied numerically. At the same time this insulator is gapless, with the gap also decaying exponentially with $\sqrt{L}$ but with the coefficient $C / 2$. Unlike the Mott-glass phase or a Bose-glass phase, which we will discuss below, the random-singlet insulator is characterized by a diverging density of states at zero energy and hence by a divergent compressibility $(\kappa)$ and superfluid susceptibility $\chi_{s}$. The former $\kappa=d n / d \mu$, in the spin language is the response to a field $\sigma^{z} \delta \mu$. Similarly, $\chi_{s}$ defined as the response to the perturbation $\delta\left(\hat{a}+\hat{a}^{\dagger}\right)$, with $\hat{a}$ being a bosonic annihilation operator; in the spin language, corresponds to the perturbation $\sigma^{x} \delta \psi$. Since the random-singlet ground state has SU(2) symmetry, the two responses have the same form and diverge as a.

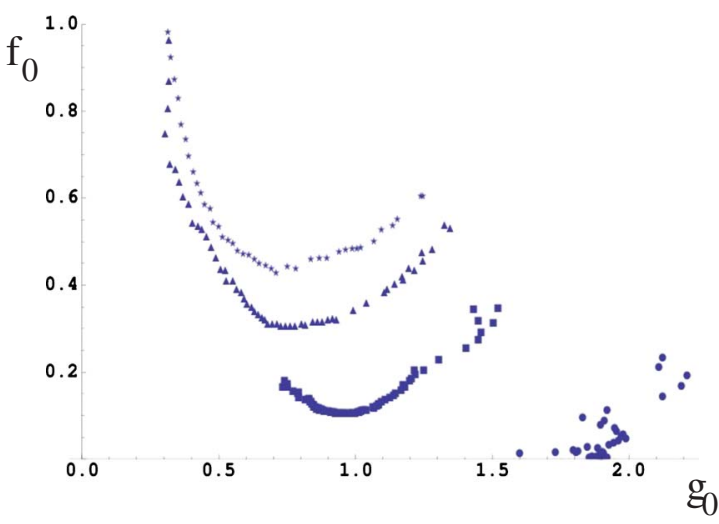

b.

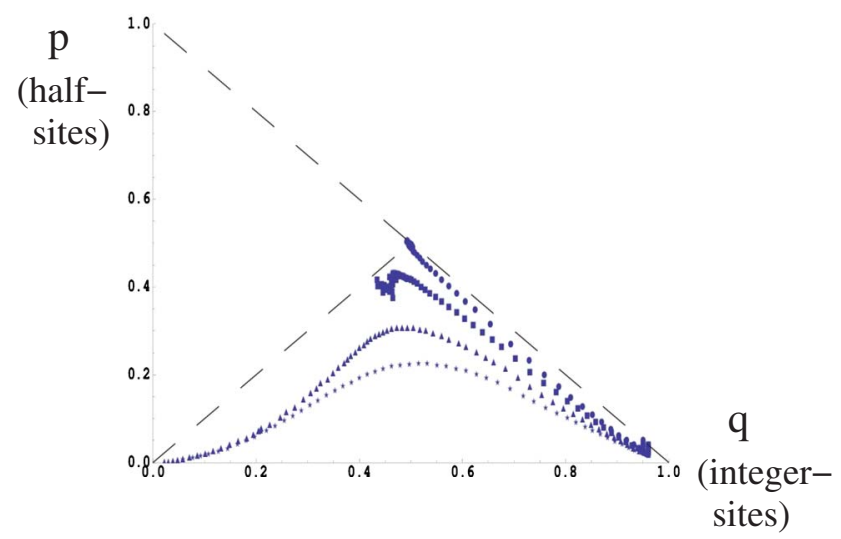

FIG. 2. (Color online) RG flows of various realizations of disorder in the (a) $f_{0^{-}} g_{0}$ plane and (b) $p-q$ plane, for chains with initial distributions characterized by $\left(\delta, p_{0}\right)=(-0.1,0.05) \quad$ (circles), $(-0.05,0.04) \quad$ (squares), $(-0.02,0.04)$ (triangles), and $(0,0.04)$ (stars). Here $p_{0}$ is the initial fraction of the half-integer $p$ sites.

$$
\kappa, \chi_{s} \sim \frac{1}{\delta \mu \log ^{3}\left(\Omega_{0} / \delta \mu\right)}, \quad \lambda \sim \frac{1}{\delta \Delta \log ^{3}\left(\Omega_{0} / \delta \Delta\right)} .
$$

As the slow decay of $g_{0}$ with $\Gamma$ suggests, indeed the randomsinglet glass has more superfluid features than the Mott glass. Both have a vanishing gap, but the Mott glass has vanishing compressibility, and its superfluid susceptibility is only finite.

\section{F. Numerical RSRG for the $p$ - $h$ symmetric state}

In order to corroborate the analytical results for the RSRG, we carried out the RG flow numerically without any simplifying assumptions. The numerics, by and large, backs the analytical results. In Fig. 2 several flow traces are given in the $f_{0}$ vs $g_{0}$ parameter space and in the $p-q$ plane. The initial distributions used consist of box distributions in the range $0.2+\delta<U<1.2+\delta$ for the charging energy and $0.2-\delta<J<1.2-\delta$ for the nearest-neighbor tunneling.

\section{PHASE DIAGRAM OF THE BOSE-HUBBARD (B-H) MODEL WITH GENERIC DISORDER}

When considering experimentally realizable models, we must also consider randomness in the chemical potential or 
random offset charges. In particular, $\bar{n}$ could have values anywhere between $-1 / 2$ and $1 / 2$. Typically, this type of disorder is very relevant. Indeed if we join two sites 1 and 2 together into a cluster then the new value of $\bar{n}_{12}$ becomes a sum of $\bar{n}_{1}$ and $\bar{n}_{2}$ modulo one (so that the result also belongs to the $(-1 / 2,1 / 2]$ interval).

To address this problem it is worthwhile review a few important insights gained from our analysis of the particlehole symmetric model. In that case the low-energy behavior was dominated by the line $p=q$ where the number of half sites with $\bar{n}=1 / 2$ was the same as the number of integer sites, with $\bar{n}=0$. At the same time we saw that the universal properties of the SF-INS (superfluid-insulator) transition with $p=q$ and $p=0, q=1$ were identical up to a factor of one half in Eq. (14), which is absent in the integer filling model.

We thus can anticipate that the fixed point governing the SF-insulator transition has a uniform distribution of $\bar{n}$ when we remove the particle-hole symmetry restrictions. But also, in analogy with the half-integer case, we can expect that the fixed point describing the SF-INS transition remains intact. On the other hand, again having the $p$ - $h$ symmetric case in mind, we expect that the distribution of $\bar{n}$ at the critical point strongly affects the properties of the insulating phase. As it turns out, the diagonal disorder plays the role of a "dangerously irrelevant variable" (as $s$ is in the analysis aboveirrelevant in the SF side of the transition but strongly relevant in the insulator side). The diagonal disorder does not change the nature of the critical and crossover behavior but it determines to which insulating phase the system will flow. In Ref. 33 the $p$ - $h$ symmetric model with only integer fillings had a Mott-glass insulating phase. By allowing also sites with charging degeneracy $(\bar{n}=1 / 2)$ but preserving the $p-h$ symmetry, the system in its insulating phase is a randomsinglet glass. When we remove the $p$ - $h$ symmetry, we expect that the insulator becomes a Bose glass: gapless, compressible state with a diverging susceptibility to SF fluctuations.

Before going into more detailed analysis, which confirms the above assertions, we would like to comment on the similarities with the perturbative RG approach of Giamarchi and Schulz. ${ }^{28}$ In particular, they derived the following flow equations near the transition between superfluid and localized phases:

$$
\begin{gathered}
\frac{d \mathcal{D}}{d \Gamma}=\frac{9}{2}\left(K^{-1}-\frac{2}{3}\right) \mathcal{D}, \\
\frac{d\left(K^{-1}\right)}{d \Gamma}=\frac{1}{2} \mathcal{D},
\end{gathered}
$$

where $K$ is the Luttinger parameter, $\sqrt{\rho_{s} \kappa}$ and $\mathcal{D}$ is proportional to the variance of the disorder in the chemical potential: $\overline{\mu(x) \mu\left(x^{\prime}\right)} \propto \mathcal{D} \delta\left(x-x^{\prime}\right)$. We point out that in Ref. 28, Eqs. (24) and (25) are written in terms of the inverse Luttinger parameter being $K$, the inverse of the common convention which we use (see, e.g., Ref. 40). Note that there is a direct analogy between Eqs. (13) and (14) and Eqs. (24) and (25) if one identifies $K$ with $g_{0}$ and $\mathcal{D}$ with $f_{0}$.
Interestingly in Ref. 30 it was argued that the SF-INS transition described by Eqs. (24) and (25) does not belong to the KT universality class because of the first power of $\mathcal{D}$ appearing in the second of these equations, as opposed to the second power in the conventional case. This difference according to the authors lead, in particular, to the unconventional scaling of the correlation length with $K-K_{c}$ near the critical point. However, this must be a misstatement since, as we argued earlier, the substitution $\mathcal{D}=\sigma^{2}$ brings the flow equations to the conventional KT form. This change in variables should not affect the scaling. Moreover the flow equations in terms of $K$ and $\sigma$ are more natural because $\sigma$ has dimensions of the external potential and thus it (not $\mathcal{D}$ ) is analogous to the strength of the periodic potential, which drives the transition in a nondisordered case.

The similarity between the perturbative analysis of Ref. 28 and the one presented here goes even further. A simple scaling argument shows that disorder in the chemical potential is strongly relevant in both approaches. However in the language of Ref. 28 the strongly relevant part of the disorder corresponds to the forward scattering, which can be reabsorbed into the canonical smooth fluctuations of the density. It is the backward scattering or phase slips, which determine the fate of the superfluid phase. By analogy with our approach we can argue that even the smooth part of the disorder potential should become strongly relevant in the insulating regime. Thus it should play the role of a dangerously irrelevant term just as the distribution of $\bar{n}$ does in our approach. Unfortunately the pertubative RG approach becomes uncontrolled in the insulating regime and this postulate cannot be reliably verified.

\section{A. RG scheme for the generic disorder B-H model}

We probe the observsations above by extending our RSRG analysis to treat arbitrary disorder: $U_{i}, J_{i}$, and $\bar{n}_{i}$ all random in the model in Eq. (1). First let us analyze the charging term while ignoring the hopping. Each site has a charge gap given by

$$
\Delta_{i}=\frac{1}{2} U_{i}\left(1-2\left|\bar{n}_{i}\right|\right),
$$

where $-1 / 2<\bar{n}<1 / 2$.

As before, we treat the model iteratively, but this time, in each step of the RG we find the largest energy scale

$$
\Omega=\max _{i}\left\{J_{i}, \Delta_{i}\right\}
$$

and eliminate it. If it is a gap, $\Delta_{i}$, then the site $i$ freezes into its lowest energy charging state. Quantum fluctuations induce an effective hopping between sites $i+1$ and $i-1$,

$$
J_{i-1, i+1}=\frac{J_{i-1} J_{i}}{2 \Omega}\left(\frac{1}{\left(1+2\left|\bar{n}_{i}\right|\right)}+\frac{1}{\left(12\left|\bar{n}_{i}\right|\right)}\right) .
$$

The last multiplier in this expression is a nonuniversal prefactor, which varies between 1 and $1 / 2$. This prefactor does not affect any universal features of the transition and we can safely set it to unity. On the other hand, if $J_{i}$ is the largest energy scale, then the sites $i$ and $i+1$ form a SF cluster with 
effective charging energy given by Eq. (3) and with a filling offset,

$$
\bar{n}_{i, i+1}=\bar{n}_{i}+\bar{n}_{2}
$$

where the last equality is defined modulo adding or subtracting one, so the the result always belong to the interval: $\bar{n}_{i, i+1} \in(-1 / 2,1 / 2]$.

\section{B. Generic case flow equations}

The ensuing flow can be quantified using flow equations for the distribution of logarithmic couplings, $\beta_{i}=\log \Omega / J_{i}$, and the joint distribution $F(\zeta, \bar{n})=f(\zeta, \bar{n}) \chi(\zeta-1+2|\bar{n}|)$, where $\zeta_{i}=\Omega / U$ and the Heaviside step function $\chi$ enforces the constraint $\Omega / \Delta>1$ or equivalently $\zeta>1-2|\bar{n}|$.

The flow equations are given by

$$
\frac{\partial g}{\partial \Gamma}=\frac{\partial g}{\partial \beta}+\left[f_{1}+g_{0}\left(1-f_{s}\right)\right] g \times\left. g\right|_{\beta=\beta_{1}+\beta_{2}}+g\left(g_{0} f_{s}-f_{1}\right),
$$

$$
\frac{\partial f}{\partial \Gamma}=\zeta \frac{\partial f}{\partial \zeta}+g_{0} f \times\left. f\right|_{\zeta=\zeta_{1}+\zeta_{2}, \bar{n}=\bar{n}_{1}+\bar{n}_{2}}+f\left(1+f_{1}-g_{0} f_{s}\right),
$$

where

$$
f_{1}=\int_{-0.5}^{0.5} d \bar{n}(1-2|\bar{n}|) f(1-2|\bar{n}|, \bar{n})
$$

is the density of sites with a large charging energy,

$$
f_{s}=\int d \zeta \int d \bar{n} \chi(\zeta-1+2|\bar{n}|) f \times\left. f\right|_{\zeta=\zeta_{1}+\zeta_{2}, \bar{n}=\bar{n}_{1}+\bar{n}_{2}} .
$$

Physically $1-f_{s}$ is the density of strong bonds connecting the sites with large onsite interaction, which are close to half filling. These sites form a cluster with $\Delta>\Omega$ and thus have to be eliminated as a spin singlet. In the equations above $\times$ implies the convolution over $\beta$ in $g \times g$ and over both $\zeta$ and $\bar{n}$ in $f \times f$.

Although the equations look somewhat obstruse, near the critical point they can be solved with the same scaling ansatz as before. Indeed, since near the transition the interactions are negligible, we can safely ignore $\delta \zeta$, which is on the order of 1 , in the convolution [Eq. (33)]. Also similarly to the particle-hole symmetric case we can expect that near the critical point the distribution of $\bar{n}$ is uniform and thus $f(\zeta, \bar{n})$ does not depend on $\bar{n}$. We then use our standard scaling ansazt,

$$
\begin{gathered}
g(\beta)=g_{0} e^{-g_{0} \beta}, \\
f(\zeta, \bar{n})=\frac{f_{0}^{2}}{1-e^{-f_{0}}} e^{-f_{0} \zeta} \approx f_{0} e^{-f_{0} \zeta},
\end{gathered}
$$

where in the last equality we used $f_{0} \ll 1$. In the same approximation of small $f_{0}$ we find that $f_{1} \approx f_{0} / 2$. Substituting the scaling ansatz into the flow Eqs. (30) and (31) and using $f_{0} \ll 1$ we immediately recover that $f_{0}$ and $g_{0}$ obey Eqs. (13) $\mathrm{f}_{0}$

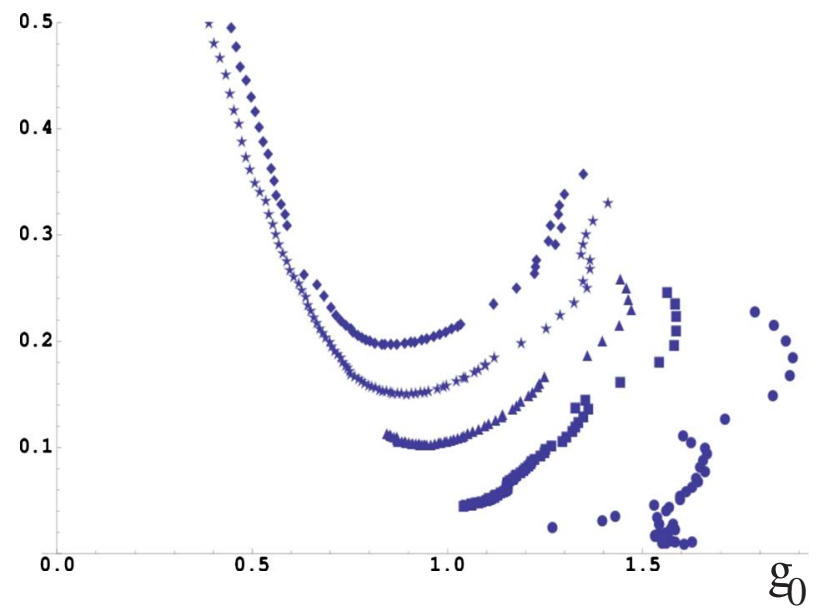

FIG. 3. (Color online) Flows in the $f_{0}$ vs $g_{0}$ plane. Initially, the interaction energy $U$ and the hopping $J$ are uniformally distributed in the range $0.2-\delta<J<1.2-\delta$ and $0.2+\delta<U<1.2+\delta$; the offset charge is also uniformally distributed between $-\Delta \bar{n}<\bar{n}<\Delta \bar{n}$. The values of $\delta$ and $\Delta \bar{n}$ for the plots shown are $\delta=-0.05, \Delta \bar{n}=0.12$ which is in the SF phase (circles), $\delta=-0.04, \Delta \bar{n}=0.08$ (squares), $\delta=-0.03, \Delta \bar{n}=0.08$ (triangles), $\delta=-0.02, \Delta \bar{n}=0.08$ (stars), and $\delta=0, \Delta \bar{n}=0.12$ (diamonds).

and (14). The latter automatically implies that the SF-IN transition in the case of generic disorder belongs to the same universality class as in the particle-hole symmetric case.

We confirm these findings performing numerical analysis of the full RG, Eqs. (30) and (31). We find a clear signature of the $K-T$ transition that is even more pronounced than before. In Fig. 3 the flows in the $f$ - $g$ parameter space are shown for three different initial conditions.

\section{Nature of the Bose glass}

We now turn to the analysis of the insulating phase at generic disorder, i.e., of the Bose glass. Even though the simple exponential form of $f(\zeta, \bar{n})$ and $g(\beta)$ does not give the exact solution to the flow equations, as we deduce from numerical analysis, it gives a very good approximation to the true distributions. For large values of $f_{0}$, Eq. (35) simplifies to $f(\zeta, \bar{n}) \approx f_{0}^{2} \exp \left(-f_{0} \zeta\right)$. It is straightforward to check that under these conditions we have $f_{1} \approx 1$ and $f_{s} \approx 4 f_{0} \exp \left(-f_{0} / 2\right)$. Then the flow elquation for $g_{0}$ becomes very simple,

$$
\frac{d g_{0}}{d \Gamma} \approx-g_{0}
$$

Such flow indicates that the Bose-glass phase is indeed intermediate between the Mott Glass where $g_{0}^{\prime} \approx-f_{0} g_{0}$ and the random-singlet insulator with $g_{0}^{\prime} \approx-g_{0}^{2}$. Physically the parameter $g_{0}$ characterizes the strength of the hoppings remaining in the system. As we argued before slow $1 / \Gamma$ decay of $g_{0}$ in the random-singlet phase resulted in the divergent density of states at zero energy and as a result in a divergent compressibility. On the other hand in the Mott glass $g_{0}$ was van- 


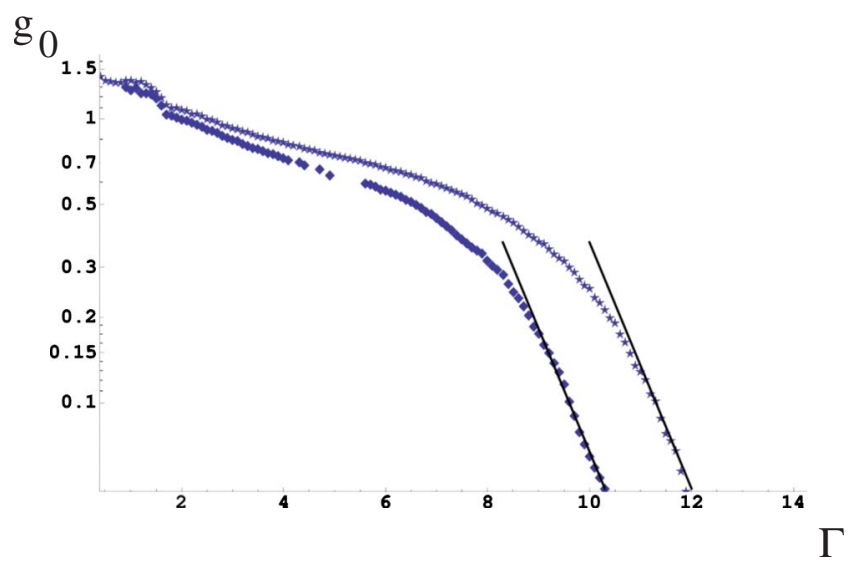

FIG. 4. (Color online) A semilog plot of $g_{0}$ vs $\Gamma$ in the Boseglass phase. The two samples are the same as the star and diamond curves in Fig. 3 with $\delta=-0.02, \Delta \bar{n}=0.08$ (stars) and $\delta=0, \Delta \bar{n}$ $=0.12$ (diamonds). The dark lines are guide to the eye and have a slope of -1 in the plot. As can be seen, the late stage of the flow of $g_{0}$ fits $g_{0} \sim e^{-\Gamma}$ very well.

ishing very rapidly $g_{0} \sim \exp -[\exp (\Gamma)]$ and thus the corresponding Mott glass had a vanishing density of states at zero energy and vanishing compressibility. In the Bose-glass phase we have exactly intermediate behavior: $g_{0} \sim \exp (-\Gamma)$. As we will see shortly this scaling implies finite density of states at low energies and thus a finite compressibility. In Fig. 4 we plot the flows of the parameter $g_{0}$ as a function of $\Gamma$ obtained from numerical solution of the RG equations for two different insulating samples with generic disorder. As can be seen from the latter figure, the flow of $g_{0}$ vs $\Gamma$ is consistent with $g_{0} \sim \exp (-\Gamma)$.

Another interesting conclusion is coming from the fact that $f_{1} \approx 1$ is independent on $f_{0}$ (and hence $d g_{0} / d \Gamma \approx-g_{0}$ ). We remind that $f_{1} d \Gamma$ gives the probability of decimating a site when we change the cutoff scale from $\Omega$ to $\Omega(1-d \Gamma)$. It is remarkable that in the Bose-glass phase this probability is independent of $f_{0}$. On the contrary, the probability of eliminating the link is proportional to $g_{0}$ and thus vanishes at long $\Gamma$. Thus we come to the conclusion that the number of the sites remaining in the system $N$ scales exactly as the cutoff energy scale,

$$
N \approx \kappa \Omega,
$$

which indicates uniform density of localized states in the insulating state. As we will see shortly the parameter $\kappa$ plays the role of the compressibility. It is interesting to note that $\kappa$ discontinuously changes across the phase transition. Indeed very close to the transition the number of sites remaining in the system is given by

$$
N(\Gamma) \sim N_{0} \exp \left(-g_{0} \Gamma\right) \sim \exp (-\Gamma) .
$$

This behavior is correct for length scales shorter than the correlation length $\xi \sim \exp (1 / \sqrt{A})$, where $A$ is the tuning parameter appearing in Eq. (15), $A \rightarrow 0$ corresponding to the critical point. After that we should use the flow equations valid in the insulating regime where $N \sim \exp \left(-f_{1} \Gamma\right) \sim \exp (-\Gamma)$. So we see that the scaling
$N \sim N_{0} \exp (-\Gamma)$ works very well. We thus conclude that the ratio $N(\Gamma) / \Omega(\Gamma)$ goes to a constant, which is independent of A.

\section{Compressibility and SF susceptibility}

The easiest way to see that $\kappa$ is indeed the compressibility is to map the renormalized array of clusters into a spin-1/2 chain. Since deep in the insulating phase the displacement $|\bar{n}|$ is close to $1 / 2$, the local interaction strengths, $U_{i}$, is mostly quite large and obeys $U_{i}>\Omega$. This implies that we could retain only the two lowest charing states, which we the spin-up and spin-down states of an effective spin $1 / 2$ degree of freedom,

$$
n_{i}=\frac{1}{2}+\hat{s}^{z}
$$

In this picture the gap $\Delta_{i}=U(1-2|\bar{n}|)$ plays the role of the external magnetic field along the $z$ axis $h_{i}^{z}$. The hopping $J_{i}$ is in turn maps to the $x y$ coupling between the neighboring spins.

Let us first determine the distribution function of $h^{z}: H\left(h^{z}\right)$ assuming that $f(\zeta, \bar{n})$ is given by Eq. (35) with $f_{0} \gg 1$,

$$
\begin{gathered}
H\left(h^{z}\right) \approx f_{0}^{2} \int_{-1 / 2}^{1 / 2} d \bar{n} \int_{1-2|\bar{n}|}^{\infty} d \zeta e^{-f_{0} \zeta} \delta\left[\left|h^{z}\right|-\frac{\Omega}{\zeta}(1-2|\bar{n}|)\right] \\
\approx \frac{1}{2 \Omega} \chi\left(\Omega-\left|h^{z}\right|\right)
\end{gathered}
$$

which is just a uniform distribution.

The compressibility of the insulating phase is given by the $z$-field susceptibility of the spin chain. The latter is easily shown to be twice the probability density of $H\left(h^{z}=0\right)$. Thus the compressibility is

$$
\kappa=\frac{\partial n}{\partial \mu}=N \frac{\partial s^{z}}{\partial h_{\mathrm{ext}}^{z}}=2 N H\left(h^{z}=0\right)=\frac{N}{\Omega},
$$

where $h_{\mathrm{ext}}^{z}$ is the infinitesimal external magnetic field along the $z$ axis. Indeed, this is the result advertised in Eq. (37).

The superfluid susceptibilty $\chi_{s}$ is obtained as the response of the spin chain on a small magnetic field in $x$ direction. Note that in the Bose-glass phase the coupling between different sites is vanishingly small and thus $\chi_{s}$ can be derived by considering an isolated site, which is described by a spin 1/2 Hamiltonian,

$$
\mathcal{H}_{i}=h^{z} \hat{s}_{i}^{z}+h_{\mathrm{ext}}^{x} \hat{\hat{s}}^{x}
$$

A straightforward calculation yields that the average magnetization along the $x$ axis is

$$
\overline{\left\langle\hat{s}^{x}\right\rangle}=\frac{1}{2} \frac{h_{\mathrm{ext}}^{x}}{\sqrt{\left(h_{\mathrm{ext}}^{x}\right)^{2}+\left(h^{z}\right)^{2}}} .
$$

Thus the susceptibility is 


$$
\chi_{s}=N \int_{\left|h_{\mathrm{ext}}^{x}\right|}^{\Omega} \frac{d h^{z}}{2 \Omega} \frac{1}{\left|h^{z}\right|}=\frac{N}{2 \Omega} \log \frac{\Omega}{\left|h_{\mathrm{ext}}^{x}\right|}=\frac{\kappa}{2} \log \frac{\Omega}{\left|h_{\mathrm{ext}}^{x}\right|} .
$$

Obviously $\chi_{s}$ diverges as $h_{\mathrm{ext}}^{x} \rightarrow 0$. Thus we find that the Bose-glass phase is characterized by divergent $\rho_{s}$ and finite $\kappa$ in agreement with Ref. 30.

\section{COMPRESSIBILITY, STIFFNESS, AND LUTTINGER PARAMETER IN THE SUPERFLUID PHASE AND AT CRITICALITY}

Let us now focus on the properties of the superfluid phase we find. The superfluid phase is associated with the formation of a superfluid cluster that spans the chain. The cluster consists of all the original bare sites that were not decimated due to their charging energies. These surviving sites obey the Hamiltonian,

$$
\mathcal{H}_{\text {eff }}^{\mathrm{SF}}=\sum_{i}\left[\frac{1}{2} \widetilde{J}_{i}\left(\varphi_{i+1}-\varphi_{i}\right)^{2}+\frac{1}{2} U_{i} \hat{n}_{i}^{2}\right],
$$

where $\varphi_{i}$ and $\hat{n}_{i}$ are the phase and number operators of the surviving cites, and $U_{i}$ are the charging energies of each bare site. The $\widetilde{J}_{i}$ are harmonic couplings between the surviving sites, which are the result of the decimation of a strong bond (marked with a tilde since they can get renormalized by intervening charge-blockaded sites) as we now explain. When deriving the $\mathrm{RG}$ equations, we eliminated the strongest bonds iteratively, by setting the sites they connected into phase-coherent clusters, which implies replacing the strongest Josephson couplings with a harmonic coupling,

$$
-J_{i} \cos \left(\varphi_{i+1}-\varphi_{i}\right) \rightarrow \frac{1}{2} J_{i}\left(\varphi_{i+1}-\varphi_{i}\right)^{2}
$$

We then approximated the cluster to be phase coherent,

$$
\tilde{\varphi} \approx \varphi_{i} \approx \varphi_{2} .
$$

This strong approximation is sufficient for obtaining the flow equations but we need to allow intracluster fluctuations it in order to discuss the properties of the superfluid phase.

The stiffness and the compressibility of the superfluid phase are given in terms of the parameters $\widetilde{J}_{i}$ and $U_{i}$ in the effective Hamiltonian (45), which describes the proliferating superfluid cluster. The compressibility is given by

$$
\kappa=\frac{1}{L} \frac{1}{U_{\mathrm{SF}-\text { cluster }}}=\frac{1}{L_{i \in \mathrm{SF}}} \sum_{i},
$$

where $L$ is the total length of the chain. The inverse superfluid stiffness is similarly obtained as

$$
\frac{1}{\rho_{s}}=\frac{1}{L} \sum_{i \in \mathrm{SF}} \frac{1}{\widetilde{J}_{i}}
$$

Note that the simple expression for the stiffness owes to the fact that the fixed-point Hamiltonian (45) is harmonic.
Therefore the stiffness suffers no further renormalization by quantum fluctuations and it is the same as in the classical model (see Ref. 33). We will now proceed to calculate the average compressibility, stiffness, and Luttinger parameter of the superfluid $K \equiv \pi \sqrt{\kappa \rho_{s}}$.

\section{A. Differential equation for the inverse-charging energy}

The compressibility given by Eq. (48) can be calculated in a rather straight forward way within the RG scheme outlaid in the previous sections. The variable $\zeta$ in the RG scheme is specifically designed to keep track of the cluster compressibilities. We recall the RG flow Eq. (9) for the distribution function $f(\zeta)$,

$$
\begin{aligned}
\frac{d f(\zeta)}{d \Gamma}= & (1+\zeta) \frac{\partial f(\zeta)}{\partial \zeta}+g_{0} \int d \zeta_{1} d \zeta_{2} \delta\left(\zeta-\zeta_{1}-\zeta_{2}-1\right) \\
& \times f\left(\zeta_{1}\right) f\left(\zeta_{2}\right)+f(\zeta)\left(f_{0}+1-g_{0}\right) .
\end{aligned}
$$

The solution to this equation will allow us to compute the compressibility from the average value of $\zeta$ as $\kappa=\bar{\zeta} / \Omega$.

To obtain a differential equation directly for the average compressibility (inverse-charging energy) we move to the Laplace-transformed representation: $\quad F(\eta)=\int_{0}^{\infty} e^{-\eta \zeta} f(\zeta)$, which obeys

$$
\begin{aligned}
\frac{d F(\eta)}{d \Gamma}= & -f_{0}+F(\eta)(\eta-1)-\eta \frac{\partial F(\eta)}{\partial \eta}+g_{0} F(\eta)^{2} e^{-\eta}+F(\eta) \\
& \times\left(f_{0}+1-g_{0}\right) .
\end{aligned}
$$

We now use that

$$
\bar{\zeta}=-\left.\frac{\partial F(\eta)}{\partial \eta}\right|_{\eta \rightarrow 0}
$$

to obtain

$$
\frac{d \bar{\zeta}}{d \Gamma}=\bar{\zeta}\left(f_{0}+g_{0}-1\right)-1+g_{0} .
$$

The inverse-charging energy is given by Eq. (48) in which an extra factor of $\Omega$ appears. Adding it on we obtain

$$
\frac{d \gamma}{d \Gamma}=\gamma\left(f_{0}+g_{0}\right)-\left(1-g_{0}\right) / \Omega
$$

where $\gamma=\bar{\zeta} / \Omega$.

\section{B. Flow equation for the stiffness}

Calculation of the stiffness requires a slight extension of the RG scheme. The method described thus far did not include a cluster variable which stores the internal stiffness. In other words the RG scheme does not keep track of the internal sum over $1 / J_{i}[$ Eq. (49)] within the proliferating clusters. 
Fortunately, such a variable can easily be included by extending the cluster distribution function $f(\zeta)$ to a joint distribution $f(\zeta, \chi)$, where

$$
\chi=\sum_{i \in \text { cluster }} \frac{1}{\widetilde{J}_{i}}
$$

is a variable designed to keep track of the superfluid stiffness of the clusters. Each time two clusters are joined in the RG flow by a large bond $J=\Omega$, the variable $\chi$ of the joined cluster is given by

$$
\chi_{12}=\chi_{1}+\chi_{2}+\frac{1}{\Omega}=\chi_{1}+\chi_{2}+\frac{1}{\Omega_{0}} e^{\Gamma} .
$$

The flow equation for $f(\zeta, \chi)$ is a straight forward extension of Eq. (50),

$$
\frac{d f(\zeta, \chi)}{d \Gamma}=(1+\zeta) \frac{\partial f(\zeta, \chi)}{\partial \zeta}+g_{0} \int d \zeta_{1} d \zeta_{2} \delta\left(\zeta-\zeta_{1}-\zeta_{2}-1\right) \int d \chi_{1} d \chi_{2} \delta\left(\chi-\chi_{1}-\chi_{2}-\Omega_{0}^{-1} e^{\Gamma}\right) f\left(\zeta_{1}, \chi_{1}\right) f\left(\zeta_{2}, \chi_{2}\right)+f(\zeta, \chi)\left(f_{0}+1-g_{0}\right)
$$

This is a rather complicated equation for the joint distribution of cluster stiffness and charging energy. However it can be greatly simplified if we are interested only in the average of the stiffness. The latter can be calculated by integrating Eq. (57) with respect to $\zeta$ and taking its Laplace transform with respect to $\chi: S(\lambda)=\int_{0}^{\infty} d \zeta \int_{0}^{\infty} d \chi e^{-\lambda \chi} f(\zeta, \chi)$. This yields

$$
\begin{aligned}
\frac{d S(\lambda)}{d \Gamma}= & -\tilde{f}(0, \lambda)-S(\lambda)+g_{0} S(\lambda)^{2} e^{-\lambda \cdot 1 / \Omega_{0} \exp \Gamma}+S(\lambda)\left(1+f_{0}\right. \\
& \left.-g_{0}\right),
\end{aligned}
$$

where

$$
\tilde{f}(0, \lambda)=\int d \chi e^{-\lambda \chi} f(\zeta=0, \chi) .
$$

Again using the fact that

$$
\bar{\chi}=-\left.\frac{\partial S(\lambda)}{\partial \lambda}\right|_{\lambda \rightarrow 0},
$$

we obtain

$$
\frac{d \bar{\chi}}{d \Gamma}=\bar{\chi}\left(f_{0}+g_{0}\right)+g_{0} / \Omega+\left.\frac{\partial \tilde{f}(0, \lambda)}{\partial \lambda}\right|_{\lambda \rightarrow 0},
$$

where we used $\Omega=\Omega_{0} \exp (-\Gamma)$ We note that the only difference between Eq. (54) and (60) is in the subleading term, $g_{0} / \Omega$ above, and $\left(g_{0}-1\right) / \Omega$ in Eq. (54). There is also the last term in Eq. (60), which should be negligible and negative.

\section{Differential equation for the length of a superfluid cluster}

The differential equation for the typical length of the clusters $^{33}$ is given by

$$
\frac{d \ell}{d \Gamma}=\ell\left(f_{0}+g_{0}\right) .
$$

It is interesting to note that this equation is the same, at the leading order, as the equations, derived above, for the sums of inverse-charging energies $\sim \bar{\zeta}$ and the sum of inverse Josephson couplings $\bar{\chi}$ within a cluster.

When calculating the stiffness and compressibility using the flow equations for a particular cluster, as illustrated above, we need to renormalize until the size of a SF cluster is that of the entire chain,

$$
\ell_{\Gamma}=L
$$

Therefore the compressibility

$$
\kappa=\frac{1}{L} \frac{1}{U_{\mathrm{SF}-\text { cluster }}}=\frac{\bar{\zeta} / \Omega}{\ell_{\Gamma}}
$$

and the inverse stiffness

$$
\frac{1}{\rho_{S}}=\frac{1}{L} \sum_{i \in \mathrm{SF}} \frac{1}{J_{i}}=\frac{\bar{\chi}}{\ell_{\Gamma}}
$$

always tend to a number as $\Gamma \rightarrow \infty$.

\section{Compressibility and stiffness at the critical point}

Let us assume that we start sufficiently close to the critical point so that the distributions $f(\zeta, \Gamma)$ and $g(\beta, \Gamma)$ already converged to the universal forms characterized by $f_{0}(\Gamma)$ and $g_{0}(\Gamma)$ [see Eqs. (34) and (35)]. In Sec. III D we found the explicit flow of these functions at criticality $g_{0}-1 \approx 2 / \Gamma$ $+2 / \Gamma^{2}$ and $f_{0} \approx 4 / \Gamma^{2}$ (that is, the flow on the separatrix). These flows start at some initial value $\Gamma_{0}$ which characterizes the bare disorder distributions of the microscopic system. The larger is $\Gamma_{0}$ the wider is the disorder distribution in $J_{i}$.

Combining this with flow equation for $\zeta[\mathrm{Eq}$. (53)] we find

$$
\frac{d \bar{\zeta}}{d \Gamma}=\bar{\zeta}\left(\frac{2}{\Gamma}+\frac{6}{\Gamma^{2}}\right)+\frac{2}{\Gamma} .
$$

This has the solution for large $\Gamma$, 


$$
\bar{\zeta} \approx C_{\zeta} \cdot \Gamma^{2} \exp [-6 / \Gamma]
$$

with $C_{\zeta}$ being a constant, which we obtain from initial conditions. We know that for sufficiently large $\Gamma_{0}, \bar{\zeta}_{0}=\frac{1}{f_{0}^{(0)}}=\frac{1}{4} \Gamma_{0}^{2}$. This implies

$$
\bar{\zeta}=\frac{1}{4} \Gamma^{2} \exp [-6 / \Gamma]
$$

and $C_{\zeta}=1 / 4$.

Similarly, for $\bar{\chi}$ we get

$$
\bar{\chi} \approx \frac{1}{\Omega} \frac{1}{6} \Gamma^{2} \exp [-6 / \Gamma]\left(C_{\chi}-\exp [6 / \Gamma]\right),
$$

where the second term in the brackets comes from the $g_{0}$ term in the equation for $\chi$. The constant $C_{\chi}$ can also be obtained from boundary conditions. At the onset $\bar{\chi}_{0}=0$ since we start with bare sites and only after some RG we get the sum of $1 / J$ to grow. This implies

$$
C_{\chi}=\exp \left[6 / \Gamma_{0}\right] .
$$

By the same token, the solution of Eq. (61) at criticality is

$$
\ell_{\Gamma}=\ell_{0} e^{\Gamma} \frac{\Gamma^{2}}{\Gamma_{0}^{2}},
$$

where $\ell_{0}$ is of order 1 .

Putting our results in the definitions of $\rho_{s}$ and $\kappa$, we obtain the compressibility of the critical system sending $\Gamma \rightarrow \infty$,

$$
\kappa=\frac{\bar{\zeta} / \Omega}{\ell_{\Gamma}}=\frac{\Gamma_{0}^{2}}{4 \Omega_{0} \ell_{0}}
$$

and the inverse stiffness,

$$
\frac{1}{\rho_{s}}=\frac{\bar{\chi}}{\ell_{\Gamma}}=\frac{\Gamma_{0}^{2}}{6 \Omega_{0} \ell_{0}}\left(\exp \left[6 / \Gamma_{0}\right]-1\right) .
$$

The energy scale for both the stiffness and the compressibility is given by $\Omega_{0}$, the initial energy scale of the problem. Both also tend to constants along the critical flow line.

\section{E. Luttinger parameter at criticality}

By multiplying Eqs. (70) and (71) we obtain the Luttinger parameter of the SF cluster,

$$
K^{2}=\pi^{2} \kappa \rho_{s}=\frac{3 \pi^{2}}{2} \frac{1}{\exp \left[6 / \Gamma_{0}\right]-1} .
$$

Indeed we find that it is a constant along flows on the critical manifold, which is independent of the initial energy scale $\Omega_{0}$. On the other hand this result is clearly not universal since it depends on $\Gamma_{0}$.

As mentioned above, $\Gamma_{0}$ parameterizes the strength of the bare bond disorder distribution. For a given system on the critical manifold, the larger is $\Gamma_{0}$ the broader is the system's initial distribution of both $J$ and $1 / U$. We can therefore interpret Eq. (72) as stating that at strong disorder, the Luttinger parameter required to stabilize a superfluid phase depends on the disorder strength. A larger Luttinger parameter is needed the more disordered is the system. This statement is clearly different from the situation at weak disorder, for which Giamarchi and Schulz had predicted a transition at a universal value of the Luttinger parameter. ${ }^{27,28} \mathrm{We}$ shall comment on the relation between these two limits in the discussion below.

\section{DISCUSSION}

\section{A. Comparison with the weak disorder limit}

Using the real-space RG approach, we obtain a consistent picture both of the possbile insulating phases of the random Bose-Hubbard model but also of the transition from the superfluid to them. The seminal work of GS (Refs. 27 and 28) obtained a description of what seems to be the same transition in terms of a perturbative RG in weak randomness- the opposite limit to our starting point. We now ask: how do these two scenarios, or descriptions, correspond to each other? Now that we obtained our result for the Luttinger parameter at criticality, Eq. (72), we can address this question.

One of the central results of Ref. 28 is the universality of the Luttinger parameter at the transition,

$$
K_{c}^{(\mathrm{GS})}=\frac{3}{2} .
$$

Since GS considered the anomalous dimension of what is essentially a phase-slip operator, the universality of $K$ at the transition was deduced from the fact that when $K>3 / 2$, phase slips are irrelevant. Since in weak-randomness phase slips are clearly the most relevant operators, the vanishing of their scaling dimension implies criticality. Also, the generality of the GS approach and the self-averaging of the SF phase $^{53}$ implies that phase slips turn relevant when $K=3 / 2$ even for strong disorder.

At strong randomness, however, we find that a different type of disturbance of the superfluid phase can disorder it. In the real-space RG analysis grains with large charging energies are decimated, implying that a whole grain becomes isolated from the rest of the chain. This process is equivalent to a phase-slip dipole happening around the grain. Phase-slip dipoles consist of a phase slip and an antiphase slip happening simultaneously at neighboring positions in the chain. In the week coupling limit, these dipoles are not enough to degrade the superfluidity since they do not produce a voltage drop. But when the disorder is strong, the dipoles, or equivalently, the blockaded insulating sites, suppress tunneling across the lattice, as we find from our analysis.

For sufficiently strong disorder, the Luttinger parameter at which blockaded sites destroy superfluidity, i.e., the critical Luttinger parameter, is given by Eq. (72),

$$
K=\pi \sqrt{\frac{3}{2} \frac{1}{\exp \left[6 / \Gamma_{0}\right]-1}} .
$$

For the $p$ - $h$ symmetric case considered in Sec. III and $K$ $=\pi \sqrt{2 /\left[\exp \left(4 / \Gamma_{0}\right)-1\right]}$ for the commensurate case, with $\bar{n}_{j}$ $=0$. As explained below Eq. (72), $\Gamma_{0}$ is a measure of the 
initial disorder of the system. Thus, $K$ grows monotonically with the disorder, and exceeds the universal GS value of $K$ $=3 / 2$ at intermediate values of $\Gamma_{0} \sim 3$. This implies that the transition we find takes over the universal GS transition at a finite disorder since we find that the breakdown of superfluidity occurs at $K>3 / 2$, the transition happens well into the region where single phase slips are irrelevant, and thus they do not modify the critical properties of the model, and can be safely ignored. This also justifies our procedure of SF cluster formation as outlined in Eq. (46). It is interesting to note that $\Gamma_{0} \sim 3$ corresponds to a charging distribution which is peaked at about $4 / \Gamma_{0}^{2} \Omega_{0} \sim 1 / 2 \Omega_{0}$, as obtained by plugging $\Gamma_{0}$ into Eq. (11).

Our conclusion is that at finite randomness the critical fixed point of the RSRG takes over (Fig. 5). When this happens, universality of the Luttinger parameter at the transition is lost. Since the transition we are describing is still a Kosterlitz-Thouless-type transition, many properties of the weak-randomness transition and strong-randomness transition are shared. One can argue that the Luttinger-parameter universality lost at strong disorder morphs into a different universlity - that of the exponent with which the distribution of $J$ vanishes at small energies, which is $g_{0}-1 \rightarrow 0^{+}$at criticality.

An outstanding question is how the weak-randomness phase-slip driven transition changes into the transition we find at strong disorder. One possiblity is that the two scenario continuously morph into each other. Yet another more exciting possiblity is that our analysis is equivalent to the calculation of the scaling dimension of an operator different from single phase slips and that such an operator becomes relevant at sufficiently strong disorder at Luttinger parameters $K$ $>3 / 2$. Therefore it causes a break down of superfluidity before phase slips become relevant.

Another important difference between the perturbative approach of GS and our results is that GS assume that the diagonal disorder is Gaussian and fully characterized by its variance while the off-diagonal disorder is weak and irrelevant. On the contrary, in the strong-randomness approach, we see that transition corresponds to a wide power-law distribution of tunneling amplitudes. Standard replica methods are not applicable to this type of disorder distribution and thus it is not surprising that the jump we find in $K$ is different. The appearance of broad power-law distribution of links in $1 \mathrm{D}$ is not surprising. There is always a finite chance of encountering a large insulating cluster separating two superfluid regions, which effectively blocks the tunneling between superfluids. This is a special property of 1D systems. In Ref. 33 we demonstrated that this is indeed the case for a simple toy model. The real-space RG just reflects this property of 1D systems. Thus if disorder is not very small so that weak links necessarily occur with finite probability, we believe that our scenario of the SF-IN transition to be more plausible than GS scenario of weak disorder. However, the final resolution of this question is currently beyond reach of both the RSRG and the GS analysis since it is concerned with the intermediate randomness regime. Probably this question can be addressed numerically.

\section{B. Real-space RG limits of validity}

In the above discussion we contrast the limits of strong and weak disorder, which begs the question of where we set the boudary between the two. As we shall argue, the RG procedure is well defined not only when the coupling distributions are of the infinite-randomness type ${ }^{36}$ but also near the axes of the $f_{0}-g_{0}$ parameter space, where $g_{0}<1$ [which yields an infinite-randomness type $\rho(J)]$ or where the charging energy has a very broad distribution, $f_{0} \ll 1$.

To obtain our results we have used essentially two renormalization-group steps. The first was a site decimation due to a large charging gap. This step assumes that the onsite charging gap $\Delta_{i}=U_{i}\left|1-2 \bar{n}_{i}\right|$ is much bigger than the neighboring Josephson coupling: $\Delta_{i} \gg J_{i}, J_{i-1}$ and it produces an effective tunneling between the neighboring sites, $J_{i-1, i+1}$ $=c J_{i} J_{i+1} / \Delta_{i}$, with $c<1$ of order unity. If the strong mismatch between $\Delta$ and $J$ is not fulfilled, we need to include higher orders in the perturbation theory, which will give rise to longer range hopping of order $\left(\tilde{J} / \Delta_{i}\right)^{n}$, with $\tilde{J}=\exp (\overline{\log J})$ the geometric average of the Josephson coupling (we assume that $f_{0} \ll 1$ and that $\Delta_{i} \gg \Delta_{j}$ for $j \neq i$ ). Even in cases of moderate disorder, as is obtained near the fixed point, where $\rho(J) \sim J^{g_{0}-1}, \widetilde{J}$ is quite small: $\widetilde{J} / \Delta \sim e^{-1 / g_{0}}$, which even at $g_{0}$ $=2$, far from the critical point, is $\sim 0.6$ implying that charge fluctuations are highly localized, and are unimportant, so long that $\rho_{J}$ has some support near $J=0$. Such tails in $\rho(J)$ are guaranteed by the Griffiths effects induced by occasional strong charging sites as described above.

The second RG step we used was a strong bond decimation. As already mentioned in Sec. III D, this RG step neglects plasma excitations within the resulting cluster. $\mathrm{Ne}$ glecting chemical-potential disorder (which only makes this perturbation step better), the effect of the plasma modes is to produce a Debye-Waller factor on the order of $\sim e^{-0.5 \sqrt{U / J}}$ in the tunneling between the cluster and its neighbors. We have neglected that in our analysis of the fixed point and its vicinity, since there $U \sim f_{0} \ll 1$, the charging energies of the constituent grains are guaranteed to be small, and the DebyeWaller suppression is negligible. Note that the decimated Josephson connection within clusters are essential to the stiffness calculation in Sec. V.

\section{EXPERIMENTAL CONSEQUENCES}

\section{A. Critical current of a finite superfluid chain}

At low-energy scales, the real-space renormalization group allows detailed knowledge of the superfluid phase. Most importantly, the effective low-energy Josephsonjunction coupling distribution is

$$
\rho(J)=g_{0} \frac{1}{J^{1-g_{0}}} .
$$

The knowledge of the Josephson distribution function allows us to make a connection with a rather simply measurable experimental property: the critical current of a chain.

Unlike the Luttinger parameter, the critical current of a bosonic chain in the absence of phase fluctuations is con- 
Lutt. Par

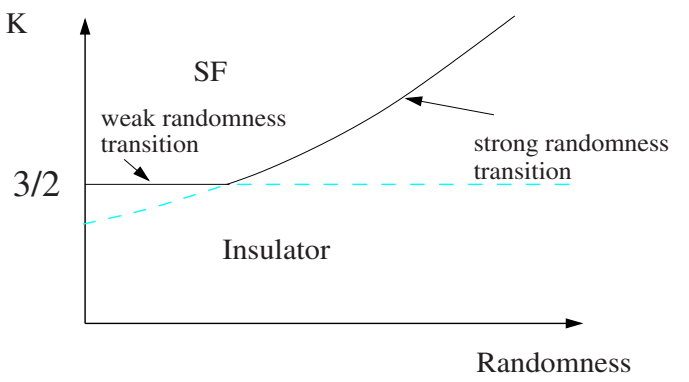

$\left(\Gamma_{0}\right)$

FIG. 5. (Color online) From all the analyses we carried out it seems that our transition does not happen at a universal value of the Luttinger parameter, but rather, at a universal value of the power law of the $J$ distribution $\left(g_{0}=1\right)$. From the discussion, it seems that there are two scenarios for the breakdown of the SF. At weak randomness it is the G-S single-vortex proliferation mechanism that first destabilizes the SF. In this range, the scenario we present would destabilize the SF at lower $K$ than single-vortex proliferation (dashed gray) and is therefore not a true boundary. At larger randomness, our scenario is the first to stabilize the SF, as it occurs at larger $K$ 's than the universal G-S value. A concequence is that the universality of the Luttinger parameter at criticality is lost.

trolled by its weakest hopping link. Given a strongly disordered bosonic chain in its superfluid phase, we can apply the real-space RG until the effective coupling distirbutions approach their universal behavior and, in particular, the distribution [Eq. (75)] for the Jospehson energies of each bond and with negligible charging effects.

Let us now calculate the scaling of the critical current on the bare length of the system. Given a particular disorder distribution, the universal distributions are obtained once the UV cutoff is $\Omega_{0}$, and only a fraction $1 / \ell$ of the chain is still active, and the chain is of length $L / \ell$. The scaling behavior of the weakest Josephson energy expectation value, $J_{\min }$, is obtained by requiring that the probability of having at least one bond with an energy $J<J_{\min }$ is of order 1 , which translates to the condition,

$$
\frac{L}{\ell} \int_{0}^{J_{\min }} \frac{d J}{\Omega_{0}} \frac{g_{0}}{\left(J / \Omega_{0}\right)^{1-g_{0}}}=1 .
$$

Carrying out the integral we obtain

$$
J_{\min } \sim\left(\frac{\ell}{L}\right)^{1 / g_{0}} .
$$

In the weak disorder regime, where $g_{0} \gg 1$ we see that the critical current is almost size independent. While at strong disorder near the transition $g_{0} \rightarrow 1+$ the critical current scales as the inverse system size. This prediction can be directly tested in experiments. Using extreme value statistics one can even find the whole Gumbel distribution of the critical current in the SF regime,

$$
P\left(J_{\text {min }}\right) \sim \frac{g_{0}}{J_{\text {min }}^{1-g_{0}}} \exp \left[-\frac{L}{\ell}\left(J_{\text {min }}\right)^{g_{0}}\right] .
$$

\section{B. Resistance at finite temperatures}

By a similar argument, we can guess the finitetemperature behavior of a disordered superfluid chain. First, we make the following simplifying assumptions: if a bond strength is $J>T$, we can neglect its finite-temperature resistance but if $J<T$, a bond will give a finite resistance $r$, which is $T$ independent. Furthermore, we ignore, for the sake of this discussion, the dependence of $r$ on $J$.

Under these simple assumptions, the resistivity $\rho$ at temperature $T$ is given by the density of bonds of strength $J<T$. Therefore,

$$
\rho \sim r \int_{0}^{T} \frac{d J}{\Omega_{0}} \frac{g_{0}}{\left(J / \Omega_{0}\right)^{1-g_{0}}}=r\left(T / \Omega_{0}\right)^{g_{0}},
$$

where, as defined above, $\Omega_{0}$ is the rough energy scale at which the chain is exhibiting the universal low-energy behavior.

In finite chains, we expect that Eq. (79) would only be valid when $T \gg J_{\min }$. Very crudely, by replacing the lower limit of the integral in Eq. (79) by $J_{\min }$ as given by Eq. (77), we obtain for $T>J_{\min }$,

$$
\rho \sim r\left|\left(\frac{T}{\Omega_{0}}\right)^{g_{0}}-\frac{\ell}{L}\right| .
$$

\section{CONCLUSIONS}

In this paper we extend the real-space $R G$ analysis of Ref. 33 to the case of noncommensurate chemical potential. We find that remarkably, the symmetry and details of the diagonal disorder are irrelevant for the SF-INS transition in a system with only onsite interactions. Nevertheless, the symmetry of the disorder completely determines the type of insulator that the system obtains. The superfluid phase will break down at a Kosterlitz-Thouless critical point and will become: (i) a gapless, incompressible, Mott glass if the chemical potential is commensurate $\left(\bar{n}_{j}=0\right)$, (ii) a gapless, compressible Bose glass with diverging superfluid susceptibility if $1 / 2<\bar{n}_{j} \leq 1 / 2$ is unrestricted, and (iii) a gapless random-singlet glass with a diverging compressiblity and superfluid susceptibilty in the case of $p$ - $h$ symmetric chemical potential $\left(\bar{n}_{j}=0,1 / 2\right)$.

An important question about our approach is its connection with the seminal work of Giamarchi and Schulz, ${ }^{27}$ we calculated the properties of the superfluid phase using the real-space RG analysis. By considering the Luttingerparameter $K$, we showed that at strong disordered the SFINS transition occurs at a finite value of $K$, larger than the universal GS value, and that the universality of the Luttinger parameter is replaced with a universality of the power-law distribution of effective hopping at low energies. The realspace RG approach is thus not complementary to the GS approach, but provides a description of the SF-INS transition at strong disorder, and allows direct access to the insulating phases, where the GS approach fails.

An interesting direction to pursue in the future is the utilization of the RSRG approach for calculation of transport 
properties and finite-temperature properties of the random 1D Bose-Hubbard chain (several works discussed recently the transport properties in the weak disorder regime and finite-temperature properties, see, e.g., Refs. 54 and 55). Such calculations could probably be done by combining our approach with that of Motrunich et al. ${ }^{56}$ The presence of very large disorder in the insulating phases should make such calculations accessible. On the other hand, they may prove difficult near the transition due to the finite randomness there.

Another outlying question is that of the correlations in the SF phase. Self-averaging indicates that the Luttinger parameter we find in Sec. V also dictates the decay of correlations in the strongly disordered superfluid phase. This, however, remains to be confirmed in direct numerical investigation of a strongly random harmonic chain. We should emphasize that due to our method for finding the Luttinger parameter, it should be consistent with the anomalous dimension of the phase-slip operator in the GS theory.

\section{ACKNOWLEDGMENTS}

We are most grateful to S. Girvin for the useful suggestion to look into the half-integer case first, and to D. S. Fisher, M. P. A. Fisher, T. Giamarchi, V. Gurarie, D. Huse, P. Le Doussal, O. Motrunich, N. Prokofe'v, and B. Svistunov for numerous discussions. A.P. acknowledges support from AFOSR YIP, NSF under Grant No. DMR-0907039, and the Sloan Foundation. G.R. acknowledges support of the Packard Foundation, Sloan Foundation and the Research Corporation. E.A. and Y.K. are grateful for support from the ISF. This work also benefited greatly from the BU visitor program (Y.K. and G.R.).
${ }^{1}$ S. Sachdev, Quantum Phase Transitions (Cambridge University Press, London, 1999).

${ }^{2}$ T. Giamarchi, Quantum Physics in One Dimension (Clarendom Press, Oxford, 2004).

${ }^{3}$ B. C. Crooker, B. Hebral, E. N. Smith, Y. Takano, and J. D. Reppy, Phys. Rev. Lett. 51, 666 (1983).

${ }^{4}$ M. H. W. Chan, K. I. Blum, S. Q. Murphy, G. K. S. Wong, and J. D. Reppy, Phys. Rev. Lett. 61, 1950 (1988).

${ }^{5}$ P. A. Crowell, J. D. Reppy, S. Mukherjee, J. Ma, M. H. W. Chan, and D. W. Schaefer, Phys. Rev. B 51, 12721 (1995).

${ }^{6}$ C. N. Lau, N. Markovic, M. Bockrath, A. Bezryadin, and M. Tinkham, Phys. Rev. Lett. 87, 217003 (2001).

${ }^{7}$ A. Bezryadin, C. N. Lau, and M. Tinkham, Nature (London) 404, 971 (2000).

${ }^{8}$ A. Rogachev and A. Bezryadin, Appl. Phys. Lett. 83, 512 (2003).

${ }^{9}$ N. Mason and A. Kapitulnik, Phys. Rev. B 65, 220505 (2002).

${ }^{10}$ N. Mason and A. Kapitulnik, Phys. Rev. Lett. 82, 5341 (1999).

${ }^{11}$ A. Kapitulnik, N. Mason, S. A. Kivelson, and S. Chakravarty, Phys. Rev. B 63, 125322 (2001).

${ }^{12}$ M. A. Steiner, G. Boebinger, and A. Kapitulnik, Phys. Rev. Lett. 94, 107008 (2005)

${ }^{13}$ Y. Qin, C. L. Vicente, and J. Yoon, Phys. Rev. B 73, 100505(R) (2006).

${ }^{14}$ G. Sambandamurthy, L. W. Engel, A. Johansson, and D. Shahar, Phys. Rev. Lett. 92, 107005 (2004).

${ }^{15}$ G. Sambandamurthy, L. W. Engel, A. Johansson, E. Peled, and D. Shahar, Phys. Rev. Lett. 94, 017003 (2005).

${ }^{16}$ B. Damski, J. Zakrzewski, L. Santos, P. Zoller, and M. Lewenstein, Phys. Rev. Lett. 91, 080403 (2003).

${ }^{17}$ J. Estève, C. Aussibal, T. Schumm, C. Figl, D. Mailly, I. Bouchoule, C. I. Westbrook, and A. Aspect, Phys. Rev. A 70, 043629 (2004).

${ }^{18}$ J. E. Lye, L. Fallani, M. Modugno, D. S. Wiersma, C. Fort, and M. Inguscio, Phys. Rev. Lett. 95, 070401 (2005).

${ }^{19}$ L. Fallani, J. E. Lye, V. Guarrera, C. Fort, and M. Inguscio, Phys. Rev. Lett. 98, 130404 (2007).

${ }^{20}$ J. E. Lye, L. Fallani, C. Fort, V. Guarrera, M. Modugno, D. S. Wiersma, and M. Inguscio, Phys. Rev. A 75, 061603(R) (2007).
${ }^{21}$ L. Fallani, C. Fort, and M. Inguscio, Advances in Atomic, Molecular, and Optical Physics (Elsevier, New York, 2008), Vol. 56 , p. 119.

${ }^{22}$ D. Clément, P. Bouyer, A. Aspect, and L. Sanchez-Palencia, Phys. Rev. A 77, 033631 (2008).

${ }^{23}$ D.-S. Lühmann, K. Bongs, K. Sengstock, and D. Pfannkuche, Phys. Rev. A 77, 023620 (2008).

${ }^{24}$ M. White, M. Pasienski, D. McKay, S. Q. Zhou, D. Ceperley, and B. DeMarco, Phys. Rev. Lett. 102, 055301 (2009).

${ }^{25}$ E. Kim and M. H. W. Chan, Nature (London) 427, 225 (2004).

${ }^{26}$ N. Prokof'ev and B. Svistunov, Phys. Rev. Lett. 94, 155302 (2005).

${ }^{27}$ T. Giamarchi and H. J. Schulz, Europhys. Lett. 3, 1287 (1987).

${ }^{28}$ T. Giamarchi and H. J. Schulz, Phys. Rev. B 37, 325 (1988).

${ }^{29}$ D.-W. Wang, M. D. Lukin, and E. Demler, Phys. Rev. Lett. 92, 076802 (2004).

${ }^{30}$ M. P. A. Fisher, P. B. Weichman, G. Grinstein, and D. S. Fisher, Phys. Rev. B 40, 546 (1989).

${ }^{31}$ T. Giamarchi, P. Le Doussal, and E. Orignac, Phys. Rev. B 64, 245119 (2001).

${ }^{32}$ E. Orignac, T. Giamarchi, and P. Le Doussal, Phys. Rev. Lett. 83, 2378 (1999).

${ }^{33}$ E. Altman, Y. Kafri, A. Polkovnikov, and G. Refael, Phys. Rev. Lett. 93, 150402 (2004).

${ }^{34}$ E. Altman, Y. Kafri, A. Polkovnikov, and G. Refael, Phys. Rev. Lett. 100, 170402 (2008).

${ }^{35}$ S. K. Ma, C. Dasgupta, and C. K. Hu, Phys. Rev. Lett. 43, 1434 (1979).

${ }^{36}$ D. S. Fisher, Phys. Rev. B 50, 3799 (1994).

${ }^{37}$ D. S. Fisher, Phys. Rev. B 51, 6411 (1995).

${ }^{38}$ K. G. Singh and D. S. Rokhsar, Phys. Rev. B 46, 3002 (1992).

${ }^{39}$ V. Gurarie, G. Refael, and J. T. Chalker, Phys. Rev. Lett. 101, 170407 (2008).

${ }^{40}$ K. G. Balabanyan, N. Prokof'ev, and B. Svistunov, Phys. Rev. Lett. 95, 055701 (2005).

${ }^{41}$ P. Sengupta and S. Haas, Phys. Rev. Lett. 99, 050403 (2007).

${ }^{42}$ G. G. Batrouni, R. T. Scalettar, and G. T. Zimanyi, Phys. Rev. Lett. 65, 1765 (1990).

${ }^{43}$ R. T. Scalettar, G. G. Batrouni, and G. T. Zimanyi, Phys. Rev. 
Lett. 66, 3144 (1991).

${ }^{44}$ J. A. Hoyos, C. Kotabage, and T. Vojta, Phys. Rev. Lett. 99, 230601 (2007).

${ }^{45}$ T. Vojta, C. Kotabage, and J. A. Hoyos, Phys. Rev. B 79, 024401 (2009).

${ }^{46}$ J. A. Hertz, Phys. Rev. B 14, 1165 (1976).

${ }^{47}$ A. J. Millis, Phys. Rev. B 48, 7183 (1993).

${ }^{48}$ G. Refael, E. Demler, Y. Oreg, and D. S. Fisher, Phys. Rev. B 75, 014522 (2007)

${ }^{49}$ G. M. Falco, T. Nattermann, and V. L. Pokrovsky, Phys. Rev. B 80, 104515 (2009).

${ }^{50}$ L. Sanchez-Palencia, D. Clément, P. Lugan, P. Bouyer, G. V.
Shlyapnikov, and A. Aspect, Phys. Rev. Lett. 98, 210401 (2007).

${ }^{51}$ D. S. Fisher and A. P. Young, Phys. Rev. B 58, 9131 (1998).

${ }^{52}$ N. Laflorencie and H. Rieger, Eur. Phys. J. B 40, 201 (2004).

${ }^{53}$ N. Prokof'ev (private communication).

${ }^{54}$ A. Petković, V. M. Vinokur, and T. Nattermann, Phys. Rev. B 80, 212504 (2009).

${ }^{55}$ I. L. Aleiner, B. L. Altshuler, and G. V. Shlyapnikov, arXiv:0910.4534 (unpublished).

${ }^{56}$ O. Motrunich, K. Damle, and D. A. Huse, Phys. Rev. B 63, 134424 (2001). 